\title{
Stromal epigenetic alterations drive metabolic and neuroendocrine prostate cancer reprogramming
}

\author{
Rajeev Mishra, ${ }^{1}$ Subhash Haldar, ${ }^{1}$ Veronica Placencio, ${ }^{1}$ Anisha Madhav, ${ }^{2}$ Krizia Rohena-Rivera, ${ }^{1}$ Priyanka Agarwal, ${ }^{1}$ \\ Frank Duong, ${ }^{1}$ Bryan Angara, ${ }^{1}$ Manisha Tripathi, ${ }^{1}$ Zhenqiu Liu, ${ }^{1}$ Roberta A. Cottlieb,, ${ }^{1,2}$ Shawn Wagner, ${ }^{2}$ Edwin M. Posadas, ${ }^{1}$ \\ and Neil A. Bhowmick $k^{1,2,3}$ \\ 1Department of Medicine, and 2Department of Biomedical Sciences, Cedars-Sinai Medical Center, Los Angeles, California, USA. ${ }^{3}$ Department of Research, Greater Los Angeles Veterans Administration, Los \\ Angeles, California, USA.
}

\begin{abstract}
Prostate cancer is an androgen-dependent disease subject to interactions between the tumor epithelium and its microenvironment. Here, we found that epigenetic changes in prostatic cancer-associated fibroblasts (CAF) initiated a cascade of stromal-epithelial interactions. This facilitated lethal prostate cancer growth and development of resistance to androgen signaling deprivation therapy (ADT). We identified a Ras inhibitor, RASAL3, as epigenetically silenced in human prostatic CAF, leading to oncogenic Ras activity driving macropinocytosis-mediated glutamine synthesis. Interestingly, ADT further promoted RASAL3 epigenetic silencing and glutamine secretion by prostatic fibroblasts. In an orthotopic xenograft model, subsequent inhibition of macropinocytosis and glutamine transport resulted in antitumor effects. Stromal glutamine served as a source of energy through anaplerosis and as a mediator of neuroendocrine differentiation for prostate adenocarcinoma. Antagonizing the uptake of glutamine restored sensitivity to ADT in a castration-resistant xenograft model. In validating these findings, we found that prostate cancer patients on ADT with therapeutic resistance had elevated blood glutamine levels compared with those with therapeutically responsive disease (odds ratio $=7.451, P=0.02$ ). Identification of epigenetic regulation of Ras activity in prostatic CAF revealed RASAL3 as a sensor for metabolic and neuroendocrine reprogramming in prostate cancer patients failing ADT.
\end{abstract}

\section{Introduction}

Despite improved detection and therapies, nearly 30,000 men continue to die of prostate cancer (PCa) in the United States and over 300,000 globally every year $(1,2)$. Currently, all therapeutic approaches for metastatic PCa involve interference of androgen signaling; however, none are curative. Examination of mechanisms of androgen signaling deprivation therapy (ADT) resistance point to a cell-autonomous process associated with expansion of a resistant clonal population, as other sensitive cells die off, and an adaptation process, in which stromal and epithelial cells coevolve to support tumor survival. The cell-autonomous processes can be a direct result of spontaneous somatic mutations favorable for an androgen-deprived environment. Evidence for stromal changes induced by ADT is associated with expression of paracrine growth factors in support of stromal-epithelial adaptation to therapeutic pressures $(3,4)$. The autocrine and paracrine processes are not mutually exclusive. Although prostatic cancer-associated fibroblasts (CAF) are not subject to somatic mutations, epigenetic changes can be used for the prediction of disease progression (5-7). We functionally differentiated CAF from their counterpart, normal associated fibroblasts (NAF), as cells that can induce tumorigenesis in nontumorigenic prostatic epithelia. NAF, also

Conflict of interest: The authors have declared that no conflict of interest exists. Submitted: January 2, 2018; Accepted: July 18, 2018.

Reference information: J Clin Invest. 2018;128(10):4472-4484

https://doi.org/10.1172/JCI99397. derived from prostatectomy tissues, are unable to induce tumorigenesis, as Cunha and colleagues have previously described (8, 9). Interestingly, the expression patterns of oncogenes and tumor suppressors in CAF have emerged as tumor promoting and tumor inhibitory, respectively. As an example, loss of the tumor suppressors phosphatase and tensin homolog (PTEN) and the TGF- $\beta$ receptor type II (TGFBR2) in fibroblastic cells is tumorigenic in breast and PCa (10-12). Similarly, the expression of oncogenes cyclin D1 (CCND1) and CMYC in the CAF has been associated with its tumorigenicity in PCa (13-15). Here, we explored how epigenetic changes can mediate oncogenic signaling in CAF and epithelial metabolic reprogramming.

Stromal-epithelial interactions can dictate cancer progression, differentiation, and even therapeutic responsiveness. The high glucose consumption of cancer cells described for many cancers is not commonly observed in PCa, as lactate generated by CAF is an important source of energy in disease progression through a familiar reverse Warburg process $(16,17)$. Interestingly, pancreatic cancer epithelia are prominently found to engulf extracellular material through a process of macropinocytosis to generate glutamine through lysosomal breakdown of serum components. Macropinocytosis is the result of activated Ras signaling endemic to pancreatic cancer, in which the glutamine generated is taken up by neighboring cells, serving to support active TCA-cycle activity $(18,19)$. Ras family proteins are small GTPases that cycle between the inactive GDP-bound and activated GTP-bound states. This cycling occurs with the help of guanine nucleotide exchange factors (RasGEFs) 
that promote activation and GTPase-activating proteins (RasGAPs) that inactivate Ras by catalyzing GTP hydrolysis. Although Ras mutations are common oncogenic drivers in many cancer types, these are infrequent in prostate tumor cells. We identified a role of Ras signaling-mediated macropinocytosis in PCa.

Oncogenic Ras promotes metabolic reprogramming of cancer epithelia by enhancing glucose uptake, glycolytic activity, and a shift to glutamine metabolism in a cell-autonomous manner. Glutamine is considered a conditionally essential amino acid, particularly for cells under stressed conditions, such as cancer. To maintain tumor growth, the carbon and nitrogen from glutamine become essential for active biosynthesis. The additional conversion of glutamine to glutamate, available through glutaminase activity (GLS and GLS2), can support the high energy needs of cancer cells, as its subsequent metabolism to $\alpha$-ketoglutarate is an entry point for the TCA cycle and oxidative phosphorylation. Elevated blood glutamate is reported to be associated with higher grade PCa (20). Nevertheless, the role of glutamine/glutamate in energetics and cellular biomass does not seem to address its association with tumor aggressiveness. It is not clear whether ADT is selective or causative of aggressive recurrent PCa. Our study explores the expanding role of ADT on stromal epigenetic changes and paracrine glutamine signaling contributing to epithelial therapeutic resistance.

\section{Results}

DNA methylome analysis revealed epigenetic silencing of RASAL 3 in prostatic $C A F$. To identify differences in the methylation status of prostate fibroblasts from human benign and PCa tissues, we performed whole-genome methylation analysis by reduced representation bisulfite sequencing (RRBS). To determine differentially methylated $\mathrm{CpG}$ sites, we analyzed, in a pairwise fashion, sequencing data from 10 primary prostatic NAF and CAF samples. The RRBS analysis led to a list of 2,000 genes that displayed differential promoter methylation in the NAF and CAF populations (Supplemental Table 1; supplemental material available online with this article; https://doi.org/10.1172/ JCI99397DS1). Among the top 20 hypermethylated promoters in CAF, 4 genes appeared to top the list and were tested: $C D 74$, RASAL3, B3GNT1, and NTRK1 (Figure 1A and Supplemental Figure $1 \mathrm{~A})$. Out of the 4 genes, $C D 74$, neurotrophic receptor tyrosine kinase 1 (NTRK1), and $\beta$-1,4-glucuronyltransferase 1 (B3GNT1), part of the glycosaminoglycan metabolism pathway, have established roles in promoting tumor progression (21-23). The RAS protein activator-like 3 (RASAL3), located at the 19p13.12 locus, is a RasGAP demonstrated to antagonize RAS signaling not previously studied with respect to its role in tumor progression. The RASAL 3 exon 2 was found to be hypermethlated in CAF $(P<$ 0.002 ; Figure 1, B and C). We selected RASAL3 as a candidate gene for further study, as promoter hypermethylation and silencing of a tumor suppressor in CAF may have a tumorigenic effect.

To confirm the methylation status of the RASAL3 promoter, methylation-specific PCR and bisulfite genomic sequencing were performed in NAF and CAF primary lines. Greater exon 2 methylation was found in the CAF compared with the NAF, as determined by methylation-specific PCR (Supplemental Figure 1B). Bisulfite sequencing of exon 2 demonstrated $73.3 \% \mathrm{CpG}$ dinucleotide methylation of RASAL3 in CAF, compared with $37.1 \%$ CpG methylation in NAF, confirming the methylome analysis (Figure 1B). Accordingly, RASAL3 mRNA expression was found to be significantly downregulated in CAF from 5 patients compared with NAF from 3 patients, as determined by quantitative reversetranscriptase PCR (RT-PCR) (Figure 1D). However, Oncomine analysis demonstrated no significant difference in RASAL3 expression in normal and PCa tumor tissues from either PRAD/ Broad or Taylor data sets $(24,25)$ Figures 1, E and F). RASAL3 was epigenetically silenced in human CAF compared with NAF, but was not differentially expressed in the epithelial compartment.

Ras signaling activation in prostatic fibroblast leads to macropinocytosis. Since the RasGAPs are negative regulators of Ras and their loss contributes to Ras pathway activation, we tested Ras activity in CAF and NAF. We found RASAL3 protein expression to be less in the primary CAF population compared with that in NAF; this was accompanied by a substantial increase in Ras-GTP (active Ras), phosphorylated c-Raf, and phosphorylated ERK (Figure 2A). Total Ras and $c$ Raf protein expression remained unchanged in NAF and CAF. We reasoned that the role of epigenetically induced Ras activation in CAF could trigger an endocytic process of macropinocytosis similar to that reported in pancreatic cancer epithelia (18). The uptake of fluorescent dextran beads (TMRdextran [dextran is $70 \mathrm{kDa}$ ]) in NAF and CAF populations was visualized. Interestingly, dextran-labeling experiments illustrated a large number of macropinosomes in $\mathrm{CAF}$, with no detectible uptake of fluorescence in NAF (Figure 2B). TMR-dextran uptake by CAF was inhibited by the macropinocytosis inhibitor 5-( $N$-ethyl$\mathrm{N}$-isopropyl) amiloride (EIPA) as well as the DNA demethylase 5-aza-2'-deoxycytidine, in support of epigenetic regulation of macropinocytosis in these fibroblasts (Supplemental Figure 2A). We further found that macropinosomes in CAF trafficked to lysosomes while undergoing a process of maturation characterized by vesicular size reduction concomitant with membrane fusion visualized by colocalization of TMR-dextran and lysotracker (Supplemental Figure 2B). To identify albumin proteolysis in CAF, we used self-quenching BODIPY dye conjugated to BSA (DQ-BSA), which emits green fluorescence following proteolytic digestion. The colocalization of TMR-dextran and DQ-BSA indicated degraded BSA was compartmentalized in macropinocytic vesicles within CAF (Figure 2C). The epigenetic silencing of RASAL3 in CAF correlated with Ras-mediated macropinocytosis and subsequent degradation of BSA.

To directly determine whether Ras signaling confers a tumorsupportive role associated with CAF, we used CRISPR/Cas9 to knock out Rasal3 in mouse prostatic fibroblasts. Compared with the expression of Cas9 fibroblasts (control), Ras activity and ERK phosphorylation were elevated in Rasal3-KO fibroblasts (Figure 2D). Rasal3-KO fibroblasts took up TMR-dextran, whereas it was undetectable in the control fibroblasts (Figure 2E). The exogenous expression of active $\operatorname{Ras}^{\mathrm{V} 12}\left(\mathrm{Gly}^{12} \mathrm{Val}^{12}\right)$ in WT mouse prostatic fibroblasts replicated the downstream ERK activation and macropinocytosis observed in CAF and Rasal3-KO cells (Figure 2, F and $G$ ). Thus, epigenetic RASAL3 silencing of prostatic fibroblasts promoted familiar signaling and macropinocytosis associated with Ras transformation (18). Conversely, PCa epithelial cell lines CWR22Rv1 and C4-2B were not found to exhibit macropinocyto- 
A

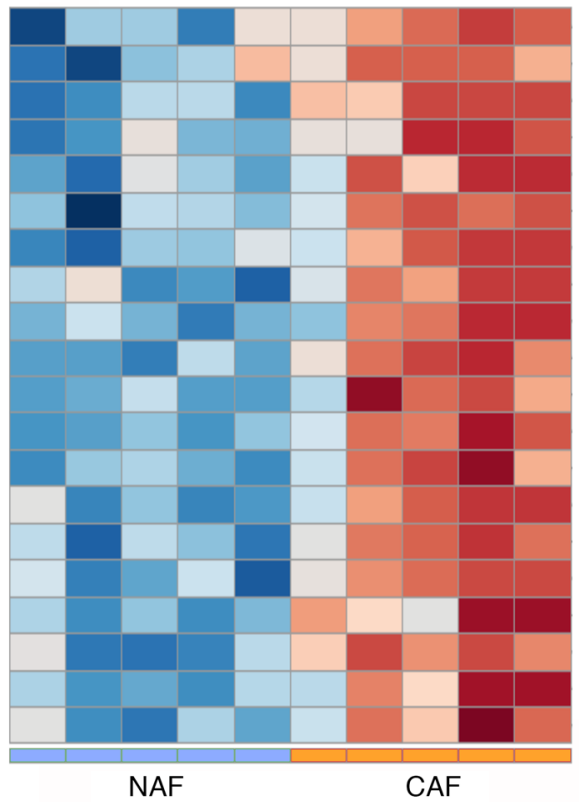

C

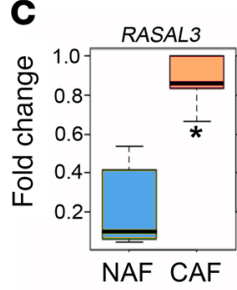

D

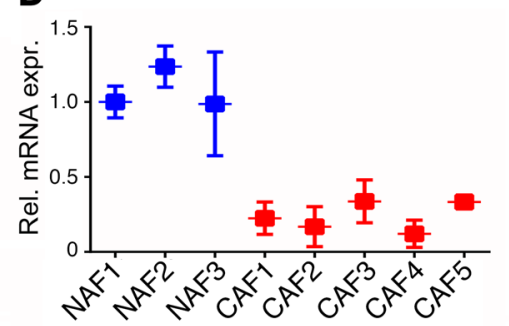

B

CALR3
TTLL10
CD74
COL9A3
AIMP1
CIITA
APOB
GPT2
FGF22
NTRK1
KIAA1161
B3GNT1
KRTCAP3
NAV1
FAM71E2
CCDC68
ALX1
RASAL3
GRIK2
HS3ST1

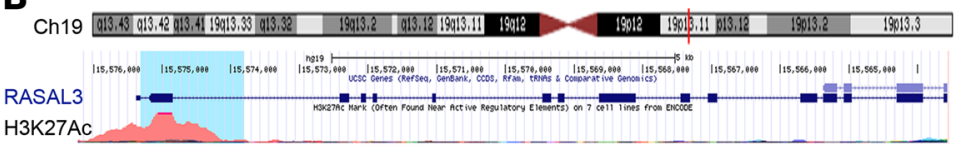

$-1$
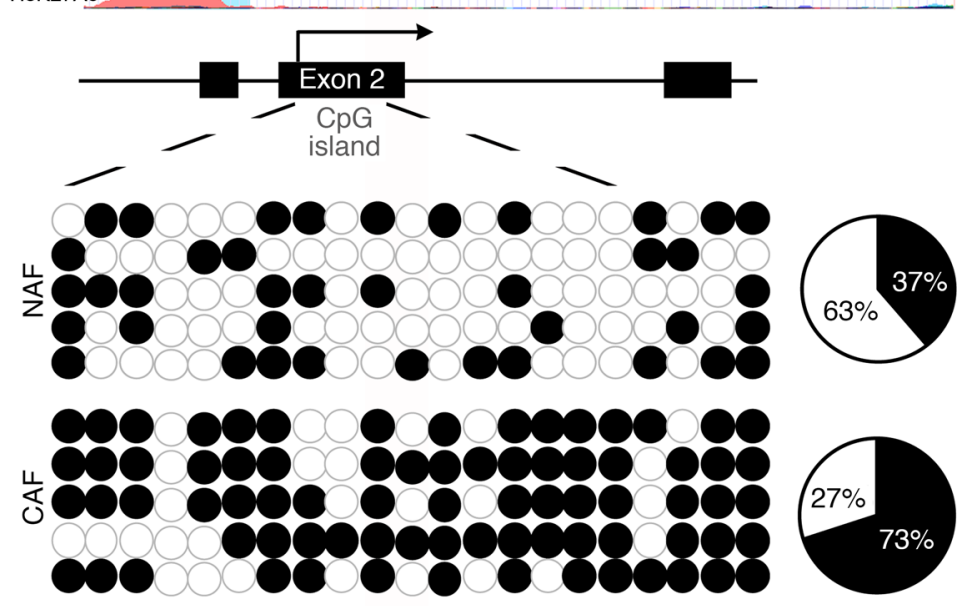

Unmethylated

Methylated
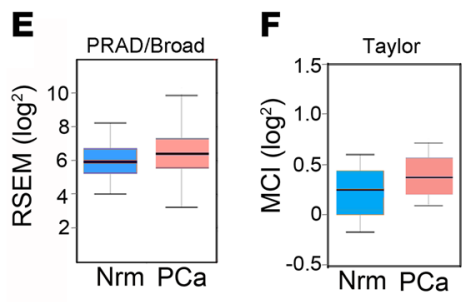

Figure 1. Epigenetic downregulation of RASAL3 in prostatic CAF. (A) Heatmap summarizing DNA methylation levels of CpG repeats (blue color indicates hypomethylation, and brown represents hypermethylation). Top 20 methylated genes significantly differentially expressed between NAF and CAF. Each column represents a fibroblast sample, and each row represents the methylation level of indicated gene $(n=5)$. (B) Top: University of California at Santa Cruz Genome Browser screen shot of the indicated region of chromosome 19 showing the positions of the RASAL3 gene, histone 3 lysine 27 acetylation, and human mRNA. Bottom: locations of predicted $\mathrm{CpG}$ sites in exon 2 by bisulfite sequencing, represented as black (methylated) or white circle (unmethylated, $n=5$ ). The number of methylated $C p G$ s divided by the total number of true CpGs analyzed is given as a percentage on the pie chart. (C) Box plots representing the difference in methylation states of the RASAL3 gene promoter between NAF and CAF as measured by RRBS analysis ( $n=5$ ). (D) RASAL3 mRNA expression in a panel of primary fibroblast was examined by quantitative RT-PCR, normalizing to GAPDH mRNA expression. rel. mRNA expr., relative mRNA expression. (E and F) Box plots show relative gene expression level $\left(\log _{2}\right)$ of the $R A S A L 3$ in normal and PCa patients from indicated data sets. Data represent mean $\pm \mathrm{SD} .{ }^{*} P<0.05$, 2-tailed Student's $t$ test.

sis, based on TMR-dextran uptake (Supplemental Figure 2C). To test the role of stromal Ras-mediated macropinocytosis on tumor development, orthotopic PCa xenograft models were used. Tissue recombinants of Ras ${ }^{\mathrm{V} 12}$-expressing fibroblasts and CWR22Rv1 epithelia were grafted into the anterior prostates of SCID mice. Two weeks later, mice were treated with vehicle control or EIPA for 1 week to suppress macropinocytosis (Figure 2H). We found that mice treated with EIPA had dramatically reduced tumor size compared with the control group. Treatment with EIPA had no significant effect on the viability of the CWR22Rv1 or WT fibroblasts, but EIPA reduced the viability of the Rasal3-KO fibroblasts $(P<$ 0.001, Supplemental Figure 3A). Thus, glutamine uptake by the tumor was necessary for its expansion.

Uptake of fibroblast-derived glutamine by PCa epithelia affects its metabolism. To establish the role of Ras on the reactive properties of fibroblasts, we tested its role on the expression of a panel of CAFdefining genes. Ras ${ }^{\mathrm{V} 12}$ fibroblasts had 2.5- to 140 -fold elevated expression of a panel of CAF marker genes compared with WT fibroblasts, inclusive of tenascin C (Tnc), Fap, Mmp1, and Mmp3 (2-way ANOVA, $P<0.0001$; Figure $3 \mathrm{~A})$. Because reactive fibroblasts are recognized as potentiating adjacent epithelial expansion, epithelial proliferation was tested in 3D cocultures with fibroblasts. PCa epithelia CWR22Rv1 and C4-2B were found to be significantly more proliferative when cultured with Ras ${ }^{\mathrm{V} 12}$ fibroblasts, compared with WT fibroblasts (Figure 3B). To determine whether a soluble factor was involved in the paracrine process, a Transwell assay was performed with Cas9 or Rasal3-KO fibroblasts in the upper chamber and CWR22Rv1 in the lower well (Supplemental Figure 3C). We found a significant proliferative induction of the epithelia when associated with Rasal3-KO fibroblasts, compared with Cas9 control fibroblasts $(P<0.0001)$. To correlate the uptake of albumin in fibroblasts and elevated Ras activity with epithelial proliferation, we tested to determine whether the stromal media affected epithelial ATP generation. Conditioned media (CM) from Ras ${ }^{\mathrm{V} 12}$ fibro- 
A

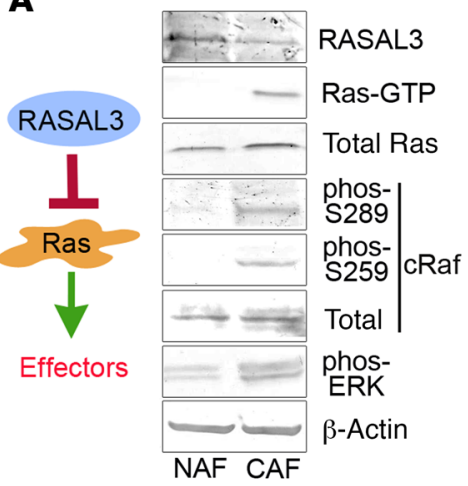

B

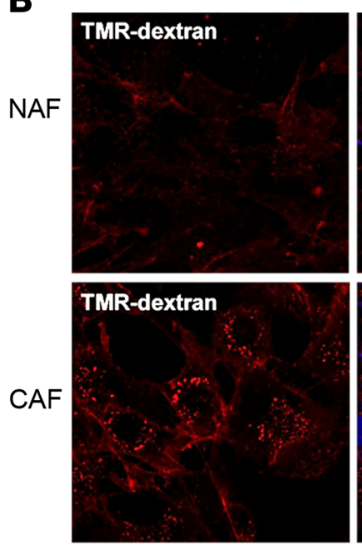

TMR-dextran

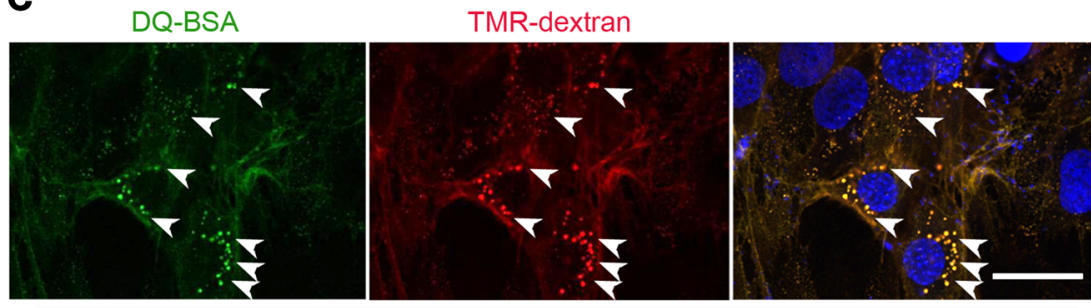

CAF DQ-BSA macropinosome labeling

E

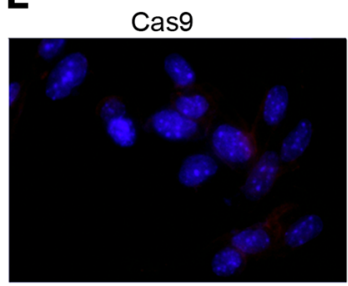

Rasal3-KO

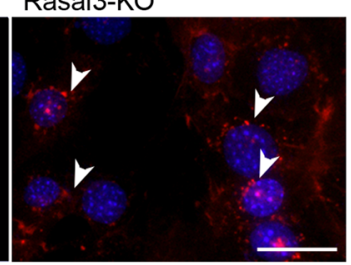

$\mathbf{F}$

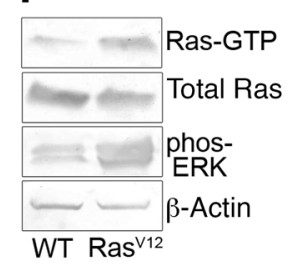

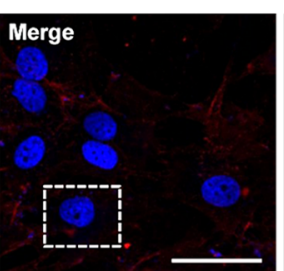

Merge

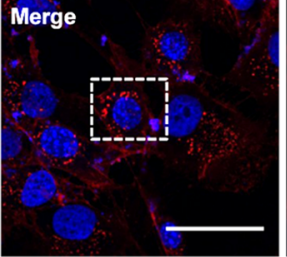

G

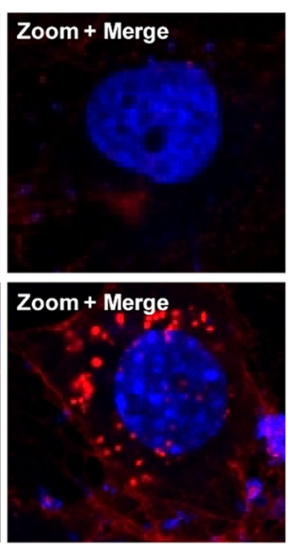

D

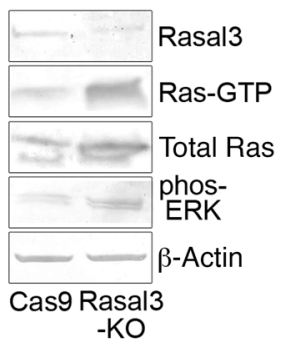

$-\mathrm{KO}$

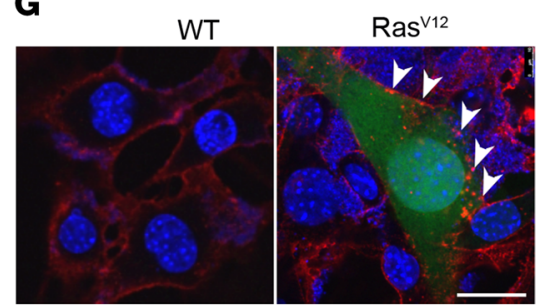

H

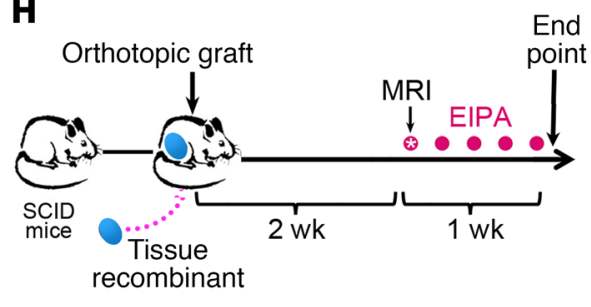

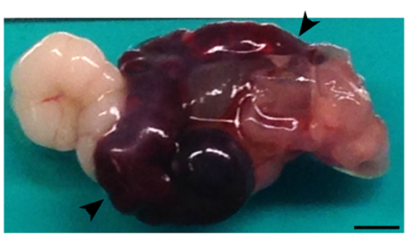

Vehicle

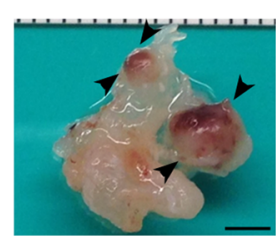

EIPA

Ras $^{\text {V12 fibro/Rv1 recombinant }}$

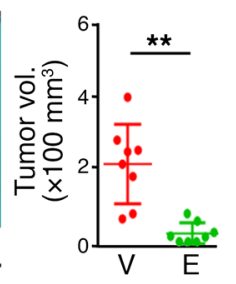

Figure 2. Ras activation stimulates macropinocytosis in prostatic fibroblasts and potentiates growth of adjacent epithelial. (A) Representative Western blots of RASAL3, Ras, and Ras effectors in prostatic human NAF/CAF $(n=3)$. See complete unedited blots in the supplemental material. (B) TMR-dextran (red) uptake by CAF shows elevated levels of macropinocytosis compared with NAF. DAPI staining (blue) identifies nuclei. (C) CAF that were coincubated with fluorescent DQ-BSA (green) and TMR-dextran (red) were fixed after 1-hour chase. The fluorescent signal emanating from DQ-BSA with TMR-dextran-positive staining (arrowheads) indicates albumin uptake by macropinosomes and subsequent breakdown. (D) Representative Western blots of RASAL3, total Ras, Ras-GTP, and phosphorylated ERK (phos-ERK) expression by Rasal3-KO and Cas9 control mouse prostatic fibroblasts are shown ( $n=3)$. (E) Representative images show TMR-dextran-positive macropinosomes (arrowheads) in Rasal3-KO prostatic mouse fibroblasts compared with Cas9. (F) Representative Western blots of mouse prostatic fibroblasts, WT and expressing Ras ${ }^{\mathrm{V} 12}$, indicate total Ras, RasGTP, and phosphorylated ERK status $(n=3)$. (G) Representative images show TMR-dextran-positive macropinosomes (arrowheads) in Ras ${ }^{\text {V12 }}$ prostatic mouse fibroblasts (expressing GFP) compared with their WT counterparts. (A-G) Representative images of 3 independent experiments are shown. (H) Schematic illustrates orthotopic tissue recombinant xenograft models of CWR22Rv1 and Ras ${ }^{\mathrm{V} 12}$ mouse fibroblasts allowed to grow for 2 weeks, followed by treatment with vehicle or $10 \mathrm{mg} / \mathrm{kg}$ EIPA $(n=8)$. Representative gross tumor images are shown with a graph of all the tumor volumes. $P$ value was calculated using 2-tailed Student's $t$ test. Data are represented as mean \pm SD. ${ }^{* *} P<0.01$. Scale bars: $30 \mu \mathrm{m}$ (B, C, E, G); 3 mm (H).

blast induced ATP production in CWR22Rv1 and C4-2B more than CM from the WT counterpart (Figure $3 \mathrm{C}$ and Supplemental Figure 4A). Similar ATP generation was observed in CWR22Rv1 in the context of CAF CM compared with NAF CM. The ATP synthase inhibitor oligomycin served as the negative control for ATP detec- tion. These results suggested that Ras-induced macropinocytosis in stromal fibroblasts confers a change in tumor-energy metabolism via a paracrine soluble factor.

As it was clear that epigenetically transformed stromal fibroblasts provided the energy needs of the epithelia, the nature of 
these metabolic changes was explored. Metabolome analysis of CWR22Rv1 demonstrated glutamine and glutamate to be significantly elevated when exposed to CAF CM compared with NAF $\mathrm{CM}(P<0.01$, Figure 3D). This was consistent with macropinocytosis of albumin, as previously reported in pancreatic cancer (26). Consequently, succinate, fumarate, and malate levels were significantly elevated in the CAF CM-treated group, compared with those in the NAF CM group, consistent with glutamine metabolism $(P<0.05)$. However, there was no significant difference in the levels of acetyl-CoA, $\alpha$-ketoglutarate, and 2-hydroxyglutarate, but there were decreased levels of citrate in the epithelia treated with CM from CAF compared with NAF. The lack of a difference in $\alpha$-ketoglutarate levels could be a result of increased flux to its metabolites (i.e., succinate, fumarate, malate). Further, there was an observed increase in levels of the nucleotide precursor aspartate $(P<0.05)$ and oxidized glutathione (GSH) $(P<0.01)$, metabolites that are downstream of glutamate metabolism (Figure $3 \mathrm{E}$ ). Compared with NAF CM, CAF CM did not significantly change reduced GSH levels, which are associated with increased mitochondrial activity and reactive oxygen in cancer cells.

In agreement with past reports of macropinocytosis-induced glutamine generation $(18,27)$, we found that, like CAF, Rasal3-KO and $\mathrm{Ras}^{\mathrm{V} 12}$ mouse fibroblasts secreted significantly more glutamine into the media than their NAF or WT counterparts (Figure 3F and Supplemental Figure 4B). To support the role of stromal-derived glutamine on epithelial proliferation, we supplemented NAF $\mathrm{CM}$ with $0.4 \mathrm{mM}$ glutamine and found it restored CWR22Rv1 proliferation to a level similar to that of CAF CM, based on direct measurement of glutamine generated by CAF (Figure 3G). Developments in magnetic resonance imaging technology have enabled glutamine/glutamate imaging with improved spatial and temporal resolution. However, its application has been relegated to glioblastoma/medulloblastoma imaging, as they are commonly Ras-driven cancers with an abundance of glutamine and glutamate in the tissue (27-29). We were able to identify the presence of glutamine/glutamate in a tissue recombination xenograft model of CWR22Rv1 and Ras ${ }^{\mathrm{V} 12}$ mouse fibroblasts. To validate the specificity of the glutamine/glutamate signal, macropinocytosis was inhibited by administering EIPA to the mice and reimaged (using simulated echo acquisition; Figure $3 \mathrm{H}$ ). Stromal fibroblastic Ras-driven macropinocytosis results in glutamine production and is utilized by cancer epithelia to promote the expansion of cancer.

To corroborate the observed epithelial accumulation of glutamine and its downstream metabolites, we tested to determine whether CAF CM regulated the expression of amino acid transporters and glutaminase expression in CWR22Rv1 and C4-2B cells. Specifically, glutamine importer SLC1A5 mRNA expression was upregulated in CWR22Rv1 $(P<0.0001)$ and C4-2B $(P<0.001)$ by CAF CM compared with NAF CM (Supplemental Figure 4C). Supplementing basal media with L-glutamine consistently increased the expression of glutamine importers SLC1A5 and SCL38A2 in both CWR22Rv1 and C4-2B cells in a time-dependent manner (Supplemental Figure 4, D-G). While the SLC38A1 transporter did not seem to be regulated by exogenous glutamine in either epithelial line, CWR22Rv1 cells expressed SLC38A4 in a glutamine-dependent manner. Once the glutamine enters the epithelia, it can undergo anaplerosis, by which glutamine is converted to glutamate by glu- taminase (i.e., GLS or GLS2) to replenish the TCA cycle. Glutamine uptake was able to promote both GLS and GLS2 mRNA expression within 6 hours of treatment in C4-2B cells (Supplemental Figure $4 \mathrm{H})$. We found GLS mRNA expression levels were also elevated in the CAF CM-treated group compared with the NAF CM-treated group in both CWR22Rv1 $(P<0.0001)$ and C4-2B $(P<0.01)$ cells (Supplemental Figure 4I). GLS2 mRNA expression was, however, downregulated in CWR22Rv1 cells $(P<0.001)$ and upregulated in C4-2B cells $(P<0.0001)$ by CAF CM treatment when compared with NAF CM treatment (Supplemental Figure 4J). These data demonstrated that glutamine taken up by the epithelia are metabolized through a seemingly positive feedback mechanism to support the elevated energy needs of cancer epithelia.

We found that glutamine uptake provided energy and mediated differentiation signaling for the prostatic epithelia. The prostatic CAF potentiated epithelial TCA flux, resulting in a mitochondrial response in CWR22Rv1 cells by the administration of exogenous glutamine (Figure 4A). Similarly, Ras ${ }^{\mathrm{V} 12}$ fibroblast CM upregulated basal oxygen consumption, ATP generation, and maximal mitochondrial respiration in CWR22Rv1 and C4-2B, compared with WT fibroblast CM (Figure 4B and Supplemental Figure 5A). Glutamine deprivation by treatment with an SLC1A5 inhibitor, L- $\gamma$-glutamyl-p-nitroanilide (GPNA), on CWR22Rv1 and $\mathrm{C} 4-2 \mathrm{~B}$ in the context of Ras ${ }^{\mathrm{V} 12}$ fibroblast $\mathrm{CM}$ restored basal respiration, ATP, and maximal respiration to that observed with WT fibroblast CM. To determine the impact of stromal-derived glutamine on epithelial proliferation, we blocked glutamine uptake and utilization with selective inhibitors in 3D cocultures of primary human CAF and CWR22Rv1 cells. We found that blocking SLC1A5 (i.e., GPNA) and GLS (i.e., bis-2-[5-phenylacetamido-1, 2, 4-thiadiazol-2-yl] ethyl sulfide [BPTES]) function significantly decreased epithelial proliferation, as determined by quantitating Ki-67 staining of EpCAM ${ }^{+}$cells through FACS analysis $(P<$ 0.05 and $P<0.001$, respectively; Figure $4 \mathrm{C}$ ). Thus, the stromal epigenetic modification resulted in glutamine production for the maintenance of ATP levels and proliferation in cancer cells. Given that $\mathrm{mTOR}$ is a nutrient sensor for glutamine, we reasoned that the same pathway may affect mechanisms of PCa differentiation $(30,31)$. We found mTOR to be activated in CWR22Rv1 cells incubated with Ras ${ }^{\mathrm{V} 12}$ fibroblast CM (Figure 4D). Moreover, expression of forkhead box M1 (FOXM1), a key signal downstream of mTOR and a critical transcription factor in multiple poorly differentiated cancer types (32-34), was upregulated in CWR22Rv1 cells incubated with Ras ${ }^{\mathrm{V} 12}$ fibroblast CM. FOXM1 was also found to interact with multiple neuroendocrine differentiation factors, as shown by STRING analysis (Figure 4E) (35). Remarkably, the incubation of CWR22Rv1 cells with Ras ${ }^{\mathrm{V} 12}$ fibroblast CM resulted in a significant increase in neuroendocrine differentiation genes shown in a heatmap (F test, $P<0.0001$; Figure $4 \mathrm{~F}$ and Supplemental Table 2). Similarly, the addition of L-glutamine alone upregulated a panel of neuroendocrine genes in CWR22Rv1 epithelia (F test, $P$ $<0.0001$ ), with the inhibition of mTOR by rapamycin restoring its expression to control levels (Figure 4G). The sufficiency for glutamine alone to induce neuroendocrine differentiation was further confirmed in C4-2B cells ( $P<0.0001$, Supplemental Figure $5 \mathrm{~B})$. Finally, we found that the knockdown of either SLC1A 5 or GLS by siRNA was able to reverse the effect of glutamine-induced neu- 
$$
\text { A }
$$
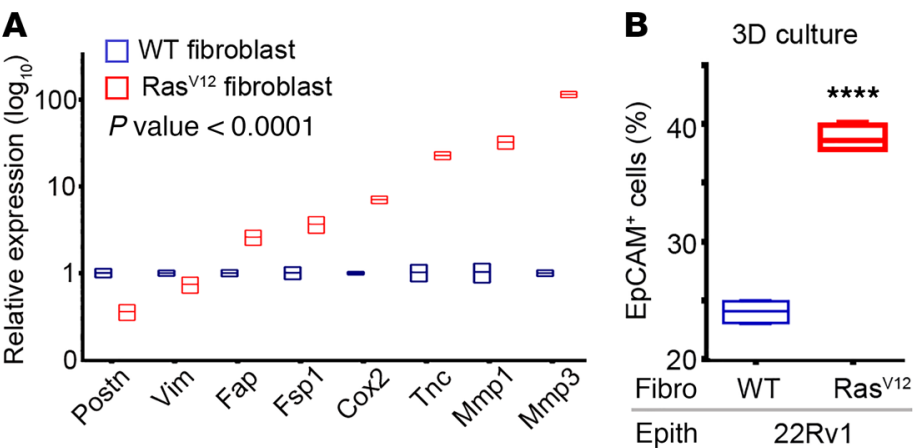

D

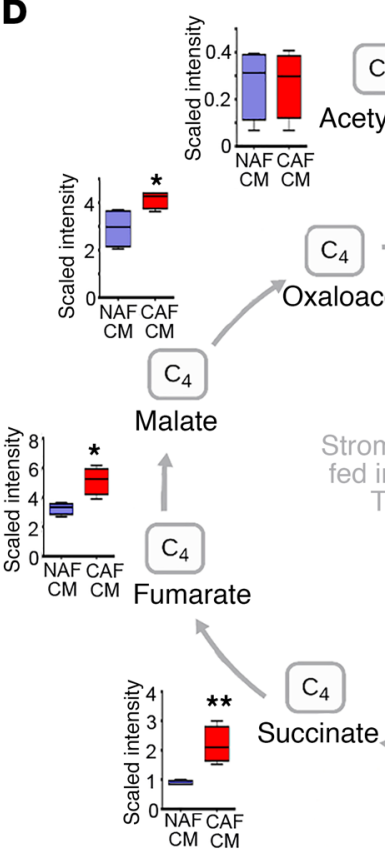

$\mathbf{F}$

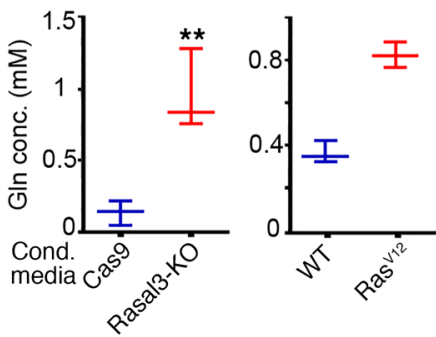

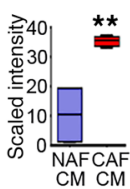

Glutamine

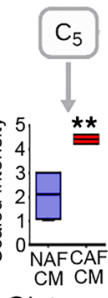

Glutamate

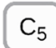
Succinyl CoA ஸे $^{\circ} \frac{\text { NAFCAF }}{\text { NAF }}$

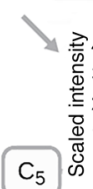

G

Stromal CM + glutamine

H

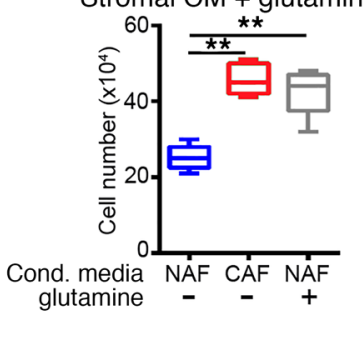

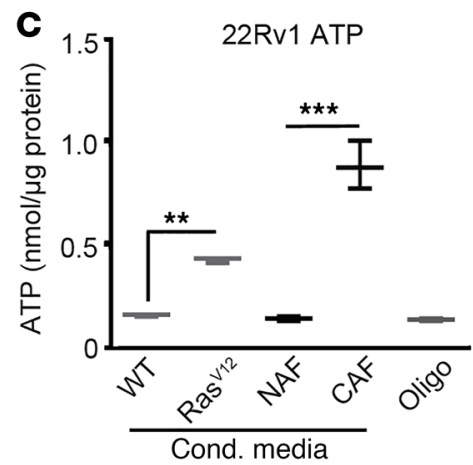

E
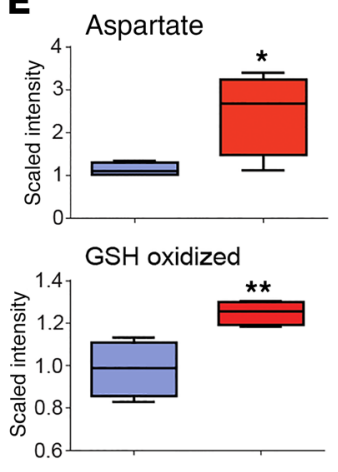

GSH reduced

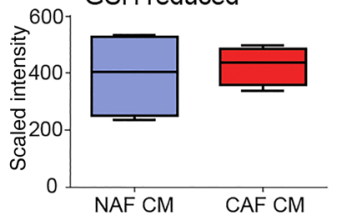

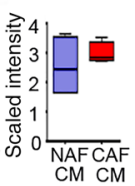

2-hydroxyglutarate

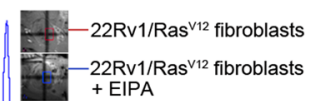
+ EIPA

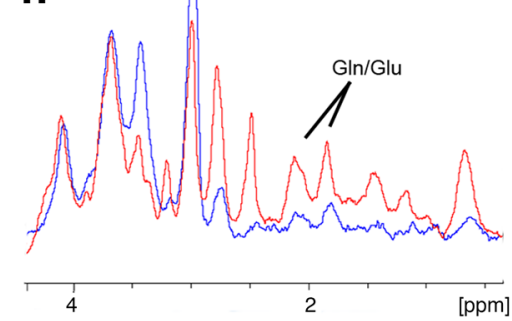

Figure 3. Ras activation in CAF drives glutamine metabolism in epithelia. (A) Relative mRNA expression levels of CAF-marker genes in WT and Ras ${ }^{\mathrm{V} 12}$ mouse fibroblasts determined by quantitative RT-PCR and analyzed using 2-way ANOVA $(n=3)$. (B) EpCAM-positive cancer epithelium was quantitated by FACS of 3D cocultures of human epithelial (CWR22Rv1) cells and WT or Ras ${ }^{\mathrm{V} 12}$ mouse fibroblasts. Statistical analysis was performed using 2-tailed Student's $t$ test $(n=3)$. (C) ATP was measured in CWR22Rv1 after incubation with CM human NAF or CAF or mouse WT or Ras ${ }^{\mathrm{V} 12}$ fibroblast or oligomycin in glutamine-free media $(n=3)$. Statistical analysis was performed using 1-way ANOVA with multiple comparisons. (D) Diagram represents the TCA cycle in CWR22Rv1 cells treated with NAF or CAF CM for 72 hours prior to metabolome analysis $(n=3)$. (E) Metabolome analysis further indicated differential flux to aspartate, oxidized GSH, and GSH reduced in CWR22Rv1 cells incubated with NAF or CAF CM. (F) Glutamine concentrations (GIn conc.) were measured in the CM from indicated mouse fibroblasts cultured for 72 hours. cond, conditioned. (D-F) Statistical analysis was performed using 2-tailed Student's $t$ test $(n=3)$. (G) CWR22Rv1 proliferation was measured by cell counting following incubation with NAF and CAF CM for 72 hours in glutamine-free media. NAF CM was supplemented with $0.4 \mathrm{mM}$ glutamine to mimic the glutamine levels expressed by CAF (see Supplemental Figure 4B). Statistical analysis was performed using 1-way ANOVA with multiple comparisons $(n=3)$. (H) Proton magnetic resonance spectroscopy data were acquired from orthotopically xenografted mice before and after EIPA administration. Spectra of the unfiltered data are superimposed using simulated echo acquisition $(n=3-4$ per group). Glu, gllutamate; ppm, parts per million. ${ }^{*} P<0.05 ;{ }^{* *} P<0.01 ;{ }^{* * *} P<0.001 ;{ }^{* * *} P<0.0001$. 
A

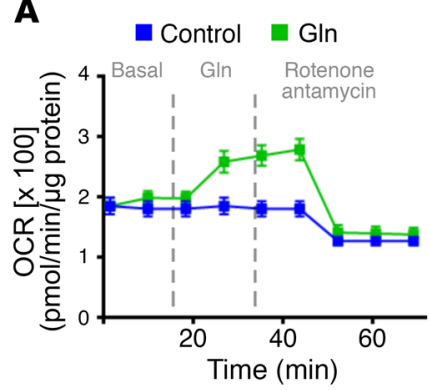

B

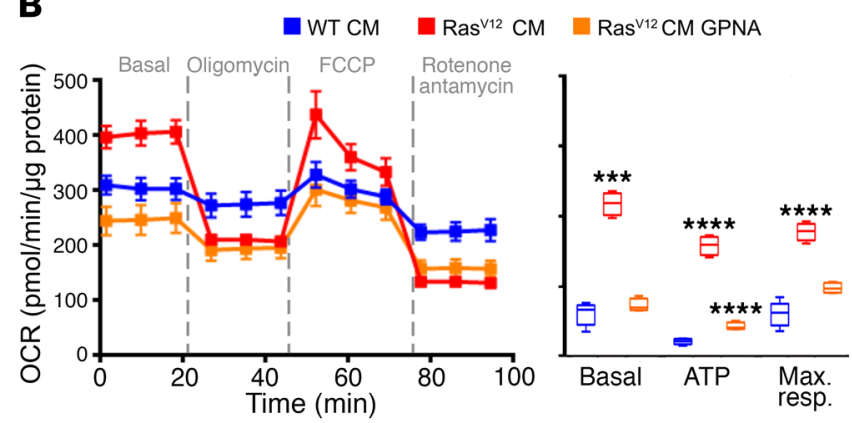

C

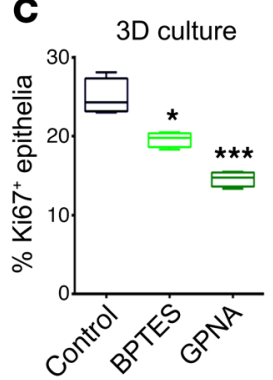

D

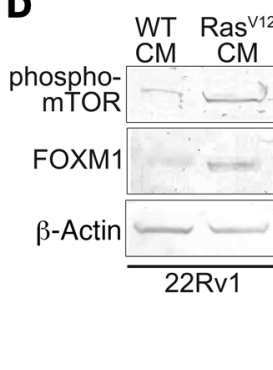

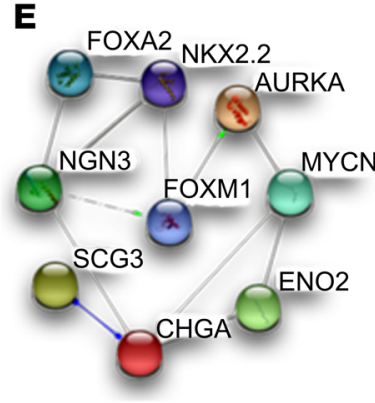
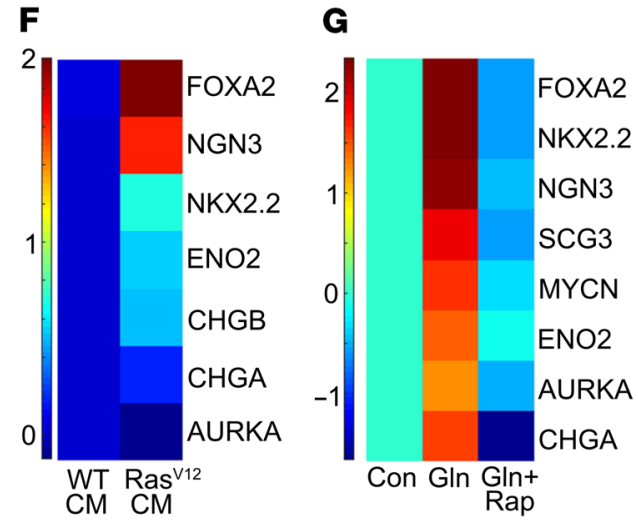

Figure 4. Stromal-derived glutamine induces mitochondrial bioenergetics and PCa neuroendocrine differentiation. (A) Kinetic OCR response in CWR22Rv1 cells exposed to $2 \mathrm{mM}$ glutamine and rotenone along with antamycin. The OCR values were normalized to total protein. (B) Bioenergetic parameters of CWR22Rv1 cells incubated with WT or Ras ${ }^{\mathrm{V} / 2}$ fibroblast CM in the presence or absence of GPNA. Left: OCR trace for all 3 groups. Right: basal OCR, ATP, and maximal respiration (max. resp.) are separately represented. (C) CWR22Rv1 cells were cocultured with WT and Ras ${ }^{\mathrm{V} / 2}$ mouse prostatic fibroblasts in 3D and treated with vehicle, BPTES, or GPNA. Box plot of epithelial proliferation was measured by FACS analysis of EpCAM+Ki67+ cells. Statistical analysis was performed using 2-tailed Student's $t$ test. (D) Phosphorylated mTOR and FOXM1 protein levels were measured in CWR22Rv1 cells incubated with either WT or Ras ${ }^{\mathrm{V} 12}$ CM for 3 days. (A-D) Representative data from 3 independent experiments are shown. (E) STRING analysis of a PCa neuroendocrine gene signature demonstrates direct or indirect interactions with FOXM1. (F) Heatmap summarizing the quantitative PCR results comparing CWR22Rv1 expression of neuroendocrine marker genes following treatment with CM from WT or Ras ${ }^{\sqrt{12}}$ fibroblasts or (G) L-glutamine (GIn) alone or L-glutamine in combination with rapamycin relative to vehicle. rap, rapamycin. Two-way ANOVA analysis indicates overall $P$ value with $\mathrm{F}$ test showing $P<0.0001$ for both experiments $(n=3 ; \mathbf{F}, \mathbf{G})$. Epithelial and stromal cells were grown in either glutamine-free DMEMF12 or RPMI media. Data are represented as mean \pm SD. ${ }^{*} P<0.05 ;{ }^{* *} P<0.001 ;{ }^{* * *} P<0.0001$.

roendocrine differentiation of CWR22Rv1 (Supplemental Figure 5C and Supplemental Table 5). Taken together, these results demonstrated that stromal glutamine was sufficient and necessary for epithelial proliferation and differentiation to a more aggressive PCa phenotype mediated by stromal Ras activity.

Epigenetic alteration of RASAL3 mRNA expression by androgen signaling affects glutamine-driven tumor progression. Neuroendocrine $\mathrm{PCa}$ is rare; however, neuroendocrine differentiation is more commonly observed as a potential adaptive response to newer, more potent ADT (ref. 36; Figure 4G and Supplemental Table 3). To explore the possible involvement of androgen signaling on prostatic stromal epigenetic alteration, we initially measured RASAL3 mRNA expression in the context of androgen and androgen receptor antagonists. As before, we found that NAF populations had greater RASAL3 mRNA expression compared with CAF (Figure 5A and Supplemental Figure $6 \mathrm{~A}$ ). The treatment with the stable androgen analog R1881 had no effect on RASAL3 mRNA expression levels in NAF; however, it markedly elevated RASAL3 mRNA expression in CAF. Conversely, androgen receptor antagonists bicalutamide and enzalutamide downregulated RASAL3 mRNA expression in NAF, yet had little effect on the already low basal mRNA expression of RASAL 3 in CAF. As evidence of epigenetic alteration of CAF by androgen signaling, we found that the demethylating agent 5aza-2'-deoxycytidine restored RASAL3 mRNA expression in CAF, similar to that found with R1881. The expression data were supported by methylation-specific PCR, demonstrating the hypermethylation of the RASAL3 promoter by bicalutamide and enzalutamide and hypomethylation by R1881, similar to that seen with 5-aza-2'deoxycytidine treatment (Supplemental Figure 6B). To quantitate methylation status of the RASAL3 exon 2 region, we performed bisulfite sequencing of CAF treated with R1881. Androgen signaling was found to prevent CpG methylation of RASAL3 by 2.6-fold over that seen with vehicle-treated CAF (Figure 5B).

To determine the role of resulting glutamine on neuroendocrine differentiation, 3D cocultures of CAF-CWR22Rv1 were treated with glutamine uptake or conversion blockers GPNA or BPTES in the context of enzalutamide. The administration of either BPTES or GPNA significantly reduced the MRNA expression of the panel of neuroendocrine biomarker genes to well below control levels (F test $P<0.0001$, Figure $5 \mathrm{C}$ and Supplemental Table 4). As neuroendocrine differentiation can potentiate PCa castrate resistance, in the next set of mouse studies, we tested the role of glutamine uptake on tumor expansion. In tissue-recombinant xenograft models, mice were either left intact or castrated, then 
A

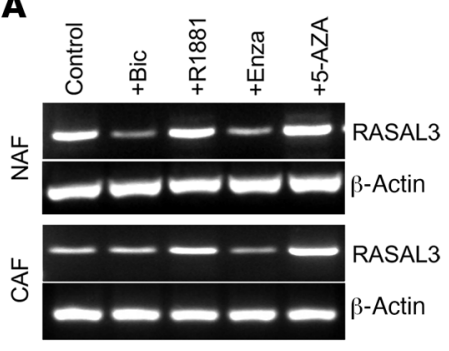

C

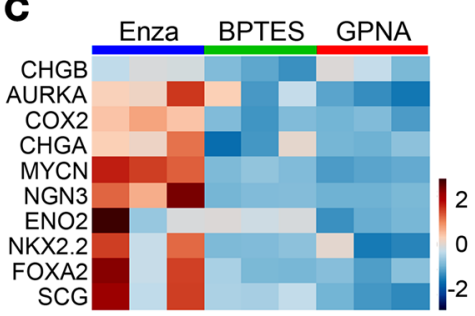

$\mathbf{E}$

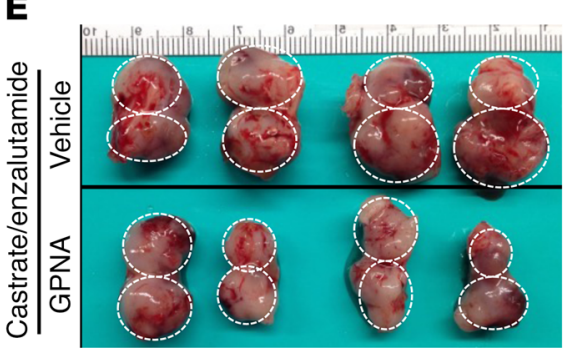

B

D
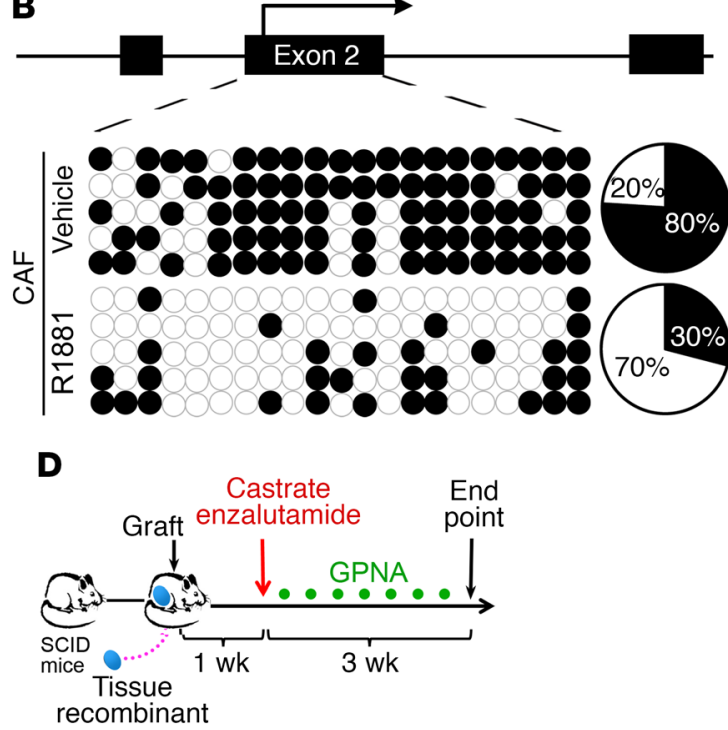

$\mathbf{F}$

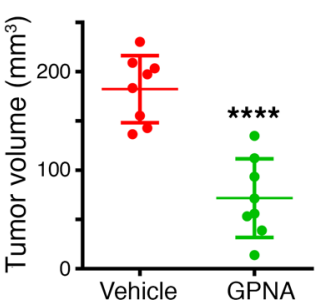

Figure 5. Androgen-mediated epigenetic regulation of RASAL3 expression in CAF determines PCa neuroendocrine differentiation and tumor growth in castrate-resistant xenograft models. (A) RT-PCR analysis of RASAL3 mRNA expression in NAF and CAF was performed following 5-day treatment with bicalutamide (Bic) $\left(10^{-5} \mathrm{M}\right)$, enzalutamide (Enza) $\left(10^{-5} \mathrm{M}\right)$, R1881 $\left(10^{-9} \mathrm{M}\right)$, or 5-azacytadine (5-Aza) $(5$ $\mathrm{mmol} / \mathrm{l})$. $\beta$-Actin was used as a loading control. Representative image of 3 independent experiments is shown. (B) Bisulfide sequencing of the RASAL3 exon 2 was performed on CAF following treatment with vehicle or R1881 for 5 days. The methylation status of the individual $\mathrm{CpC}$ dinucleotides is shown by unmethylated (white) or methylated (black) circles $(n=5)$. (C) 3D cocultures of human CWR22Rv1 with CAF were treated with vehicle, enzalutamide, BPTES, or GPNA for 3 days in glutamine-free media and subjected to quantitative RT-PCR for the expression of a neuroendocrine PCa gene panel ( $n=3$, see Supplemental Table 4). Heatmap generated relative to vehicle treatment. (D) Schematic diagram of the experimental design of castration, enzalutamide, and GPNA treatment of a castrate-resistant xenograft model. Subrenal capsule xenografts contained tissue recombinants of CWR22Rv1 and CAF. (E) Gross images of representative tumors (dashed circles) as shown on host kidneys. (F) Quantitated tumor volumes are represented as the mean \pm SD, analyzed by 2 -tailed Student's $t$ test $(n \geq 8) .{ }^{* * *} P<0.0001$.

treated with enzalutamide, followed by administration of vehicle or GPNA, as outlined in Figure 5D and Supplemental Figure 7A. In intact mice, the administration of GPNA resulted in reduced tumor volume $(P<0.001)$ and mitotic index, as determined by phosphorylated histone $\mathrm{H} 3$ immunolocalization $(P<0.0001$, Supplemental Figure 7, B-D). In the castrated, enzalutamide-treated mice, we found that these tissue-recombinant tumors expanded despite ADT, but that the added treatment with GPNA significantly reduced tumor size (Figure 5, E and F; $P<0.0001$ ). Histological analysis revealed that ADT resulted in solid sheets of cells, whereas the addition of GPNA treatment was associated with karyolytic cells (Figure 6A). In agreement with the results of the neuroendocrine gene panel, GPNA treatment reduced chromogranin A expression induced by castration and enzalutamide treatwaterfall plot illustrates that glutamine concentrations above $2 \mathrm{mM}$ corresponded to disease progression on ADT and that glutamine values at or below the threshold corresponded to treatment response (Fisher's exact test odds ratio $=$ $7.451, P=0.02)$. Compared with baseline glutamine concentrations of 0.4-0.7 $\mathrm{mM}$ in normal subjects, the PCa patients in this small cohort overall had markedly elevated glutamine concentrations (average $=1.8 \mathrm{mM})$, in agreement with previously reported elevations in glutamate levels in high-grade PCa subjects (20). These results support the concept that reduced ADT sensitivity is associated with elevated circulating glutamine concentrations.

\section{Discussion}

This study reports that ADT can induce epigenetic alteration of the stromal fibroblasts to promote $\mathrm{PCa}$ progression to a more 

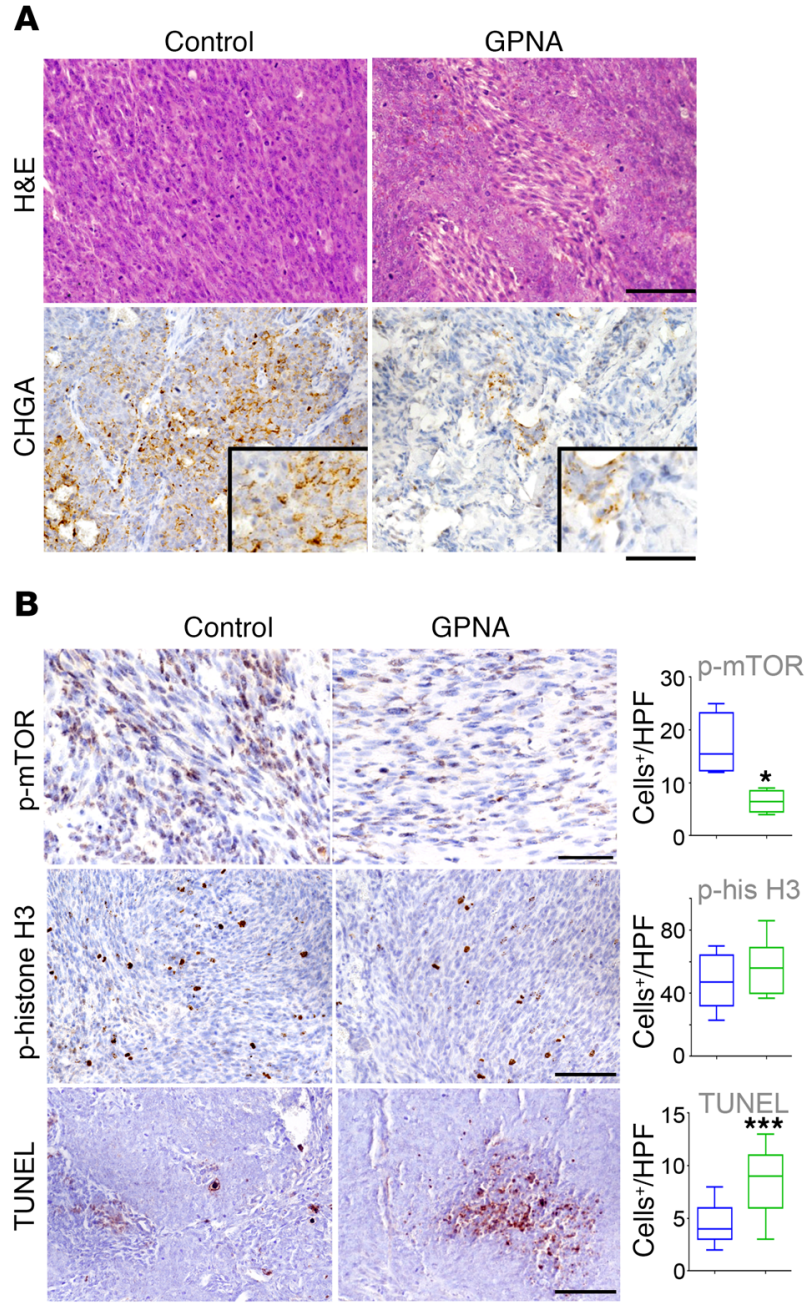

Figure 6. Glutamine uptake promotes tumor neuroendocrine differentiation and survival in the context of ADT. (A) H\&E and chromogranin A (CHCA) staining of PCa xenografts indicates regulation by glutamine uptake antagonist GPNA. (B) Representative staining for phosphorylated mTOR, phosphorylated histone $\mathrm{H} 3$, and TUNEL are shown for the PCa xenografts. Quantification of positive staining demonstrated significant differences when mice were treated with GPNA and ADT, compared with ADT alone. Scale bars: $50 \mu \mathrm{m}(n \geq 8)$. Data represent mean \pm SD. Statistical analysis was performed using 2-tailed Student's $t$ test. ${ }^{*} P<0.05$; ${ }^{* *} P<0.001$.

aggressive differentiation state. Based on our earlier report that prostatic CAF have elevated DNA hypermethylation due to epithelia-derived paracrine signals (6), we performed a wholegenome methylome analysis to reveal the silencing of a tumor suppressor, RASAL3. The outcomes of our interrogation of stromalepithelial interactions are summarized in Figure 7B, where the hypermethylation of the RASAL3 promoter in CAF induced RASdependent macropinocytosis for the lysosomal catabolism of albumin and generation of glutamine. Metabolomic profiling uncovered the role of stromal glutamine as an underlying metabolic controller facilitating cell survival and proliferation. Glutamine transporter-facilitated uptake and subsequent metabolism of stromal glutamine into epithelia for utilization by the TCA cycle and MTOR activation contribute to the energy needs of the cancer and its differentiation, respectively. This is the first demonstration, to our knowledge, of epigenetic Ras regulation in CAF consequently contributing to cancer progression. Targeting Ras signaling via RasGAPs has been a focus of many laboratories. We demonstrated that human CAFs have low expression of RASAL3 due to exon 2 hypermethylation, resulting in Ras-signaling activation. We further found that elevated Ras activity in prostatic mouse WT fibroblasts was sufficient to promote differentiation states analogous to those of the tumor-promoting $\operatorname{CAF}(8,9)$. Others have identified epigenetic silencing of RASAL isoforms associated with aberrant Ras activation in cancer epithelial subtypes $(37,38)$. RASAL1 hypermethylation, mediated by DNA methyltransferase 1 (DNMT1), is also reported to promote renal fibrosis (39). Our findings provide evidence for RasGAP gene methylation and Ras signaling in CAF. Silencing of RASAL3 contributed to downstream cRaf and ERK activation in the absence of a change in Ras expression. So the common loss of PTEN expression in PCa epithelia is not necessarily the only determinant for elevated PI3K activity in tumor tissues (40).

A key finding of the present work adds a dimension to the previously recognized epigenetic regulation by androgen signaling. Gravina et al. showed that the androgen receptor is a key regulator of DNMT expression in some PCa cell models (41). They demonstrated that PCa epithelia treated with bicalutamide or cultured in androgen-depleted medium progressively acquire greater DNMT activity compared with cells cultured under standard conditions. Conversely, $10^{-12} \mathrm{M}$ dihydrotestosterone was able to reduce DNMT activity. As prostatic fibroblasts also express the androgen receptor $(42,43)$, it was consistent with the observed RASAL3 promoter hypermethylation (Figure 5). The subsequent activation of Ras signaling suggested the testing of macropinocytosis based on the seminal identification of this process in pancreatic cancer with high Ras mutation frequency (18). The knockout of Rasal3 and overexpression of Ras ${ }^{\mathrm{V} 12}$ in WT mouse prostatic fibroblasts recapitulated the observation of macropinocytosis strikingly prevalent in primary human prostatic CAF derived from multiple patient tissues (Figures 1 and 2). Uniquely, the activation of Ras signaling in prostatic fibroblasts demonstrated elevated expression of a gene panel associated with a reactive fibroblastic state. This was not entirely surprising, since we and others have previously demonstrated the downregulation of tumor suppressors (e.g., TGFBR2, PTEN) and upregulation of oncogenes (e.g., MYC, CCND1) in CAF to have protumorigenic capacity $(10,11,13-15)$. What was not predicted, however, was that androgen deprivation further silenced RASAL3 in NAF and was coincident with acquisition of the CAF features (Figures 3 and 5). Thus, epigenetic modification of tumor-associated fibroblasts can be initiated by ADT and drive a mechanism of resistance to ADT in a paracrine manner.

Cancer cells survive and even thrive under hypoxic and in what are thought to be nutrient-deprived conditions. However, glutamine, a conditionally essential amino acid that is the most commonly depleted amino acid in solid tumors, can serve as a carbon source for the TCA cycle and as a nitrogen source for nucleotide biosynthesis (44). Like glucose, amino acids cannot cross the lipid bilayer without transporter proteins, and amino acid transporter expression is positively correlated with growth and cancer (45). We found the expression levels of glutamine transporters and glutaminase in CWR22RV1 and C4-2B cells are potentiated by glu- 
A

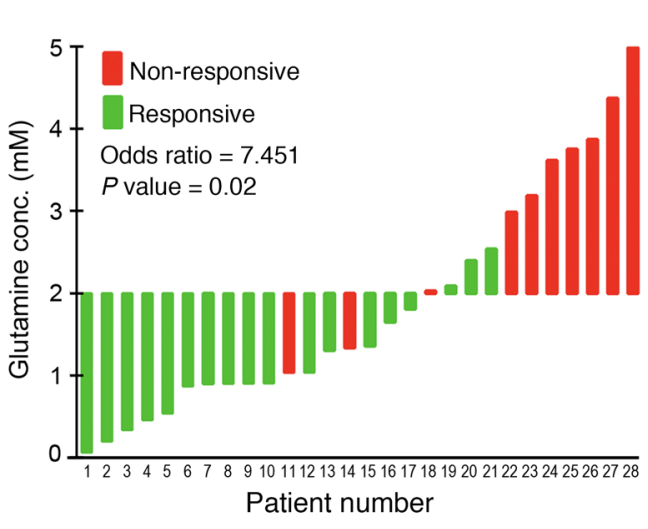

B

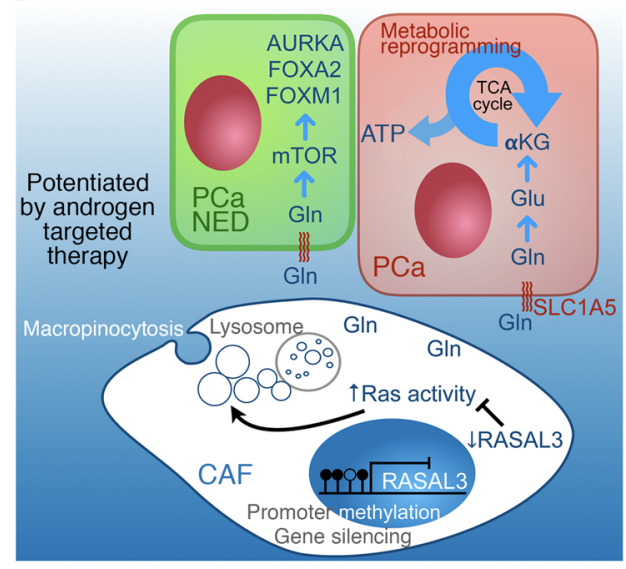

Figure 7. Sensitivity to ADT correlating with blood glutamine levels is in support of a model of epigenetic Ras activation in prostatic fibroblasts and glutamine-mediated paracrine activity on PCa epithelia. (A) Waterfall plot of plasma glutamine concentration in PCa patients on ADT correlated to therapeutic response ( $n=28$; see Table 1). Based on serum PSA detection, patients were determined to be responsive (green bars) and nonresponsive (red bars) to ADT. The threshold of $2 \mathrm{mM}$ glutamine was chosen as a determinant of ADT responsiveness. Fisher's exact test was used to determine odds ratio. (B) While RASAL3 promoter hypermethylation and gene silencing are observed in CAF compared with NAF, androgen-targeted therapy furthers this epigenetic Ras activation process that induces macropinocytosis in stromal fibroblasts for the uptake of albumin. Albumin is degraded by the lysosomes to generate glutamine and shuttled into epithelia through the glutamine transporter SLC1A5. Glutamine is converted to glutamate and $\alpha$-ketoglutarate ( $\alpha$ KC) in entering the TCA cycle in support of PCa epithelial proliferation. Clutamine also contributes to mTOR activation, leading to neuroendocrine differentiation (NED).

tamine secreted from CAF, allowing its own uptake (Supplemental Figure 4). Within the epithelia, glutamine conversion to glutamate could be metabolized into $\alpha$-ketoglutarate, aspartate, and GSH (Figure 3). The lack of observed $\alpha$-ketoglutarate accumulation was likely due to its oxidation to succinate, fumarate, and malate to address the energy needs by oxidative phosphorylation. However, glutamate conversion to significantly elevated oxidized GSH was a further indication of accumulated oxidative stress mediated by active mitochondrial electron transport (46). The prominent levels of the glutamine derivative aspartate found in the epithelia when exposed to CAF represent a key oxidized precursor for both purines and pyrimidines, which further support cell proliferation (47). Thus, the androgen-dependent switch of the tumor microenvironment that regulates glutamine acts through RASAL3 epigenetic silencing. More practically, therapeutic approaches targeting hormone signaling must consider these metabolic adaptations as a means of resistance.

The precedence of PCa neuroendocrine differentiation associated with next-generation ADT administration is a prominent manifestation of late-stage disease $(36,48)$. However, mechanisms for ADT induction of neuroendocrine $\mathrm{PCa}$ are less well understood. An example involving paracrine IL-8 derived from neuroendocrine PCa cells was found to promote neuroendocrine differentiation (49). The impact of autocrine adrenomedullin and IL-6 in neuroendocrine differentiation is also described (50, 51). Here, we defined an androgen-dependent mechanism for CAF-mediated neuroendocrine differentiation of adjacent prostatic epithelia. It is known that glutamine sensing triggers mTOR activity $(52,53)$. mTOR signaling is associated with PCa neuroendocrine differentiation $(28,54,55)$. Therefore, we reasoned that targeting glutamine metabolism may serve as a therapeutic means of limiting neuroendocrine differentiation in sensitizing PCa to ADT. Importantly, stromal glutamine was necessary and sufficient to promote PCa neuroendocrine differentiation (Figures $4-6)$. As ADT potentiated stromal glutamine secretion, combination therapy of castration/ enzalutamide and targeting glutamine uptake by cancer cells with GPNA could serve as a viable synthetic lethal approach. As with pharmacologic inhibition in a mouse model of castrateresistant disease, the knockdown of SLC1A5 limited ADT-induced neuroendocrine differentiation (Figures 5 and 6 and Supplemental Figure 5). Previous findings supported SLC1A5 as highly expressed in $\mathrm{PCa}$ and breast cancer tissues and showed that their inhibition led to reduced cell growth $(53,56)$. However, as this was the first demonstration, to our knowledge, of such a direct role of the tumor microenvironment in promoting PCa neuroendocrine differentiation, we sought to validate the findings in PCa patient blood samples. Our data suggested that plasma glutamine can be used as a prognostic marker following ADT response and development of resistance (Figure 7). The importance of the work here involves the mechanism for ADT-induced PCa neuroendocrine differentiation and further clinical validation through the measurement of plasma glutamine. Furthermore, ADT can induce epigenetic alteration of the stromal fibroblasts to promote PCa neuroendocrine differentiation in a glutamine-dependent manner.

The elevated glutamine levels detected in the blood of patients on ADT suggest that nearly any metastatic site would be exposed to this amino acid in support of elevated energy needs as well as neuroendocrine differentiation. Adjuvant ADT is commonly administered to patients with recurrent PCa. As most PCa recurrence is local, the orthotopic grafts in our studies provide a relevant basis for blocking glutamine uptake and/or metabolism. As far as distant metastasis, one could extrapolate that local tumor expansion can affect distant metastatic progression via glutamine. Future studies could include testing the role of circulating glutamine on bone and visceral metastatic progression of PCa.

\section{Methods}

Cell culture. Prostatic fibroblasts from PCa patients and mouse prostates were cultured in high-glucose DMEM-F12 supplemented with 5\% FBS, 5\% Nu-Serum, Antibiotic-Antimycotic (all from Thermo Fisher Scientific), and $10^{-9} \mathrm{M}$ testosterone (57). In order to test the tumor-inductive capacity of the primary human prostatic fibroblasts, they were xenografted with nontumorigenic BPH1 prostatic epithelia 
Table 1. Association of patient androgen-targeted therapy responsiveness and plasma glutamine levels

\begin{tabular}{|c|c|c|c|c|c|c|}
\hline Patient & Treatment & CRPC & $\begin{array}{c}\text { PSA } \\
\text { baseline }\end{array}$ & $\begin{array}{c}\text { PSA } \\
\text { on treatment }\end{array}$ & Outcome & $\begin{array}{c}\text { Glutamine } \\
(\mu \mathrm{mol} / \mathrm{l})\end{array}$ \\
\hline 1 & $A$ & Yes & 16.4 & 6.4 & Responsive & 64.6 \\
\hline 2 & $B+L$ & No & 52.2 & 0.75 & Responsive & 198.4 \\
\hline 3 & $\mathrm{~L}$ & No & 1.6 & 0.2 & Responsive & 337.5 \\
\hline 4 & B & No & 21.3 & 11.3 & Responsive & 458.3 \\
\hline 5 & L & No & 6.5 & $<0.1$ & Responsive & 539.8 \\
\hline 6 & $A+A 5$ & Yes & 9.2 & 0.1 & Responsive & 870.4 \\
\hline 7 & $B+L$ & No & 16.2 & 2.3 & Responsive & 901.9 \\
\hline 8 & $L+T$ & No & 10.7 & 0.2 & Responsive & 901 \\
\hline 9 & $E$ & No & 0.2 & 0.1 & Responsive & 903 \\
\hline 10 & $B+L$ & No & 0.2 & 0.1 & Responsive & 906.9 \\
\hline 11 & $E$ & Yes & 284.7 & 344.3 & Nonresponsive & 1035 \\
\hline 12 & $A+A 5$ & Yes & 63.5 & 116.5 & Nonresponsive & 1037.6 \\
\hline 13 & L & Yes & 7.3 & 7.8 & Nonresponsive & 1305 \\
\hline 14 & $E+R$ & Yes & 51.9 & 71.5 & Nonresponsive & 1338 \\
\hline 15 & $A+A 5$ & Yes & 218 & 522.3 & Nonresponsive & 1359 \\
\hline 16 & $E$ & Yes & 0.8 & $<0.1$ & Responsive & 1650 \\
\hline 17 & L & No & 14.3 & 0.1 & Responsive & 1809.5 \\
\hline 18 & A & Yes & 28.5 & 26.2 & Nonresponsive & 2030 \\
\hline 19 & $A+A 5$ & Yes & 4.2 & 0.8 & Responsive & 2090 \\
\hline 20 & L & No & 5.3 & 1.1 & Responsive & 2394 \\
\hline 21 & $B+L+R$ & No & 5.7 & 0.1 & Responsive & 2535 \\
\hline 22 & $B+C$ & No & 17.9 & 5.7 & Nonresponsive & 2982 \\
\hline 23 & $A+A 5$ & Yes & 61.5 & 19.8 & Responsive & 3184.4 \\
\hline 24 & $A+R$ & Yes & 17.7 & 25.6 & Nonresponsive & 3613.5 \\
\hline 25 & $B+L$ & Yes & 0.4 & 3.7 & Nonresponsive & 3751.1 \\
\hline 26 & $\mathrm{~L}$ & Yes & 0.9 & 1.2 & Nonresponsive & 3870 \\
\hline 27 & $E$ & Yes & 61.3 & 116.7 & Nonresponsive & 4369.8 \\
\hline 28 & $B+L$ & Yes & 4.4 & 0.8 & Nonresponsive & 4989 \\
\hline
\end{tabular}

$A$, abiraterone; $A 5$, apalutamide; $B$, bicalutamide; $E$, enzalutamide; $G$, goserelin; $L$, leuprolide; $\mathrm{R}$, radiation; $\mathrm{T}$, triptorelin.

the NAF and CAF samples and 200 ng of DNA was bisulfite converted by using the EZ DNA Methylation-Gold Kit (Zymo Research) according to the manufacturer's protocol. Three overlapping primers were used for amplification of the exon 2 region of RASAL3 by ZymoTaq DNA polymerase (Zymo Research) and subsequently cloned into the pCR2.1-TOPO vector (Invitrogen, Life Technologies). The primer sequences used to amplify the 3 regions were designed by using the MethPrimer program (http:// www.urogene.org/methprimer/) and are listed in Supplemental Table 6. Five clones from each sample were sequenced by using Sanger's method.

Macropinocytosis assay. Fibroblasts and PCa epithelia were grown on coverslips and serum-starved for 18 hours prior to TMR-dextran incubation $(1 \mathrm{mg} / \mathrm{ml} ; 70 \mathrm{kDa}$, FITC-labeled, Thermo Fisher Scientific) for 30 minutes at $37^{\circ} \mathrm{C}$ for macropinosome labeling (18). After removing the dextran-containing medium, cells were rinsed 5 times in cold PBS and fixed in 3.7\% formaldehyde. Coverslips in DAPI-containing mounting medium (Vectashield) were mounted onto slides. Images were captured using laser confocal scanning microscopy (Leica) and analyzed via LASX software. To determine whether the macropinosomes colocalize with lysosomes, LysoTracker Green (Thermo Fisher Scientific) was added in cells along with TMR-dextran for 30 minutes. To determine whether the internalized albumin was degraded, we utilized a selfquenching fluorescent dye, DQ-BSA $(10 \mu \mathrm{g} / \mathrm{ml}$ for 30 minutes), with which lysosomal proteases enable fluorescent visualization by confocal microscopy.

$3 D$ organotypic coculture. A modified version of the $3 \mathrm{D}$ organotypic coculture system was performed in a collagen matrix similar to that previously reported (59). Matrices were prepared by mixing 5 volumes of rat tail collagen with 2 volumes of Matrigel in 1 volume of $\times 10$ DMEM medium and 1 volume of FBS. CWR22Rv1 or C4-2B epithelia and

(gift of Simon W. Hayward, NorthShore University, Chicago Illinois, USA) under the renal capsule, as previously described $(8,9)$. The fibroblasts were termed CAF only if tumors developed within 4 weeks of grafting; otherwise, the non-tumor inductive fibroblasts were termed NAF. CWR22Rv1 and C4-2B cells (ATCC) were maintained in RPMI1640 medium with $5 \%$ FBS and $1 \%$ penicillin/streptomycin. All cultures were grown in a humidified $5 \% \mathrm{CO}_{2}$ environment at $37^{\circ} \mathrm{C}$.

DNA methylation analysis. Libraries were prepared from 200-500 ng genomic DNA digested with 60 units of TaqaI and 30 units of MspI (NEB) sequentially and then extracted with Zymo Research DNA Clean \& Concentrator 5 Kit. Fragments were ligated to preannealed adapters containing 5'-methyl-cytosine instead of cytosine according to Illumina's specified guidelines. Adaptor-ligated fragments of 150$250 \mathrm{bp}$ and $250-350 \mathrm{bp}$ in size were recovered from a $2.5 \%$ NuSieve 1:1 agarose gel (Zymo Research). The fragments were then bisulfite treated using the EZ DNA Methylation-Lightning Kit. Preparativescale PCR was performed, and the resulting products were purified for sequencing on an Illumina HiSeq. Further details of DNA methalome analysis are provided in the Supplemental Methods.

Bisulfite sequencing. Bisulfite sequencing was performed as described previously (58). Briefly, genomic DNA was extracted from primary mouse prostatic fibroblasts (WT or Ras ${ }^{\mathrm{V} 12}$ ) were combined in a 1:3 ratio in the matrix. Nylon squares were coated with collagen and placed on metal grids in a 6-well plate. Matrix plugs containing cells formed in 96 -well plates $(150 \mu \mathrm{l})$ were transferred onto the nylon squares, and medium was added to the level of the nylon mesh. Following treatments, the cells were dissociated from the matrix with collagenase and dispase for FACS analysis.

Seahorse XF-24 metabolic flux analysis. Oxygen consumption rate (OCR) (pmol/min-1) was measured with the Seahorse Bioscience XF24 extracellular flux analyzer. Cells were incubated in a $\mathrm{CO}_{2}$ free incubator for 1 hour at $37^{\circ} \mathrm{C}$ to allow for temperature and $\mathrm{pH}$ equilibration prior to loading into the XF24 apparatus. Initial assays were performed to optimize cell number, FCCP concentration, and oligomycin concentration (data not shown). We used GPNA (10 $\mu \mathrm{M}$, Sigma-Aldrich), a glutamine transporter inhibitor.

Spectral magnetic resonance for glutamine/glutamate. Chemical exchange saturation transfer effect of amine protons was used to image glutamine and glutamate, using a 9.4T micro-MRI in orthotopic prostate xenografts in mice, similar to that previously described (29). The mice were imaged before and 1 hour after intraperitoneal administration of the macropinocyosis inhibitor EIPA. 
Tissue recombination mouse models. Cell recombinants were prepared by mixing $2 \times 10^{5}$ epithelial (CWR22Rv1) cells with $6 \times$ $10^{5} \mathrm{CAF}$ or stromal cells expressing Ras ${ }^{\mathrm{V} 12}$ per site in collagen, as described before (6). Orthotopic grafting constituted the placing of the collagen plugs in the 2 anterior lobes of the prostate, and kidney grafts were placed under the renal capsule of C57BL/6 male 8-week-old mice, as described previously $(3,6)$. The mice were either left intact or castrated 1 week after grafting. The intact mice were randomly divided into 2 groups, one of which received intraperitoneal injection of $3 \mathrm{mg} / \mathrm{kg}$ EIPA (Sigma-Aldrich) or vehicle control every other day for 1 week. Castrated mice were treated with enzalutamide $(5 \mathrm{mg} / \mathrm{kg})$ either alone or in combination with GPNA $(10 \mathrm{mg} / \mathrm{kg}$ ) for 3 weeks. The tumor volumes were measured using digital calipers and calculated using the following formula: length $(\mathrm{mm}) \times$ width $(\mathrm{mm}) \times$ width $(\mathrm{mm}) \times 0.52$. Mice were sacrificed, and tumors were excised and photographed. Formalin-fixed, paraffinembedded (FFPE) xenograft tumor tissue was sectioned and stained with H\&E and also stained for chromogranin A, TUNEL, phosphorylated histone $\mathrm{H} 3$, and phosphorylated mTOR.

Statistics. RRBS BaseClear DNA-based analysis was performed for quality control, sequence processing, and mapping of sequence reads per their EpiQuest Genome-Wide Basic Service package. Sequence depth was determined per CpG dinucleotide as the number of reads for which methylation status could be determined. CpGs with a depth of less than 10 reads in either condition were excluded. Methylation status of the remaining CpGs was calculated as the percentage of methylcytosine in total cytosine. The heatmap was made using Metabo Analyst software using the top 25 genes analyzed by ANOVA. Statistical significance of differences between control and experimental groups was assessed by unpaired Student's $t$ test (2 tailed); for multiple comparisons, 1-way ANOVA was followed by Šidák's multiple-comparison tests. $P$ values reported in the manuscript were based on the Šidák cor- rection for the multiplicity problem. Repeated-measures 2-way ANOVA with post hoc analyses was also used where appropriate. $P$ values of less than 0.05 were considered statistically significant. Data presented in figure legends are represented as mean \pm SD unless otherwise stated. Statistical tests utilized are reported in the figure legends, along with the associated $P$ values.

Study approval. All mouse studies were approved by the CedarsSinai Animal Care and Use Committee. All PCa patients treated with ADT in this study, detailed in Table 1, provided informed consent for blood analysis according to requirements of the Institutional Review Board of Cedars-Sinai Medical Center.

\section{Author contributions}

RM and NAB conceived the study. RM developed methodology and provided project administration. $\mathrm{RM}, \mathrm{SH}, \mathrm{VP}, \mathrm{AM}, \mathrm{PA}, \mathrm{KRR}, \mathrm{FD}, \mathrm{BA}$, SW, and MT performed experiments. RM, FD, and ZL performed formal analysis. RM, RAG, AM, and NAB wrote the paper. NAB, RAG, and EMP supervised the study and acquired funding.

\section{Acknowledgments}

We thank the patients that contributed to this study. Matrigel was acquired from the National Cancer Institute/NIH Tumor Microenvironment Network. Spectral MR studies were performed at the Cedars-Sinai Imaging Institute with the support of the UCLA CTSI (UL1TR001881). This work was supported by grants from the National Cancer Institute (CA108646 and CA098912) and Veterans Affairs (BX001040) to NAB.

Address correspondence to: Neil A. Bhowmick, Cedars-Sinai Medical Center, 8750 Beverly Boulevard, Atrium 103, Los Angeles, California 90048, USA. Phone: 310.423.5589; Email: bhowmickn@cshs.org.
1. Siegel R, Ma J, Zou Z, Jemal A. Cancer statistics, 2014. CA Cancer J Clin. 2014;64(1):9-29.

2. Ferlay J, et al. Cancer incidence and mortality worldwide: sources, methods and major patterns in GLOBOCAN 2012. Int J Cancer. 2015;136(5):E359-E386.

3. Placencio VR, et al. Stromal transforming growth factor-beta signaling mediates prostatic response to androgen ablation by paracrine Wnt activity. Cancer Res. 2008;68(12):4709-4718.

4. Singh M, Jha R, Melamed J, Shapiro E, Hayward $\mathrm{SW}$, Lee P. Stromal androgen receptor in prostate development and cancer. Am J Pathol. 2014;184(10):2598-2607.

5. Bianchi-Frias D, et al. Cells comprising the prostate cancer microenvironment lack recurrent clonal somatic genomic aberrations. Mol Cancer Res. 2016;14(4):374-384.

6. Banerjee J, Mishra R, Li X, Jackson RS, Sharma A, Bhowmick NA. A reciprocal role of prostate cancer on stromal DNA damage. Oncogene. 2014;33(41):4924-4931.

7. Zong Y, et al. Stromal epigenetic dysregulation is sufficient to initiate mouse prostate cancer via paracrine Wnt signaling. Proc Natl Acad Sci U S A. 2012;109(50):E3395-E3404.

8. Olumi AF, Grossfeld GD, Hayward SW, Carroll PR, Tlsty TD, Cunha GR. Carcinoma-associated fibroblasts direct tumor progression of initiated human prostatic epithelium. Cancer Res. 1999;59(19):5002-5011.

9. Hayward SW, et al. Malignant transformation in a nontumorigenic human prostatic epithelial cell line. Cancer Res. 2001;61(22):8135-8142.

10. Trimboli AJ, et al. Pten in stromal fibroblasts suppresses mammary epithelial tumours. Nature. 2009;461(7267):1084-1091.

11. Bhowmick NA, et al. TGF-beta signaling in fibroblasts modulates the oncogenic potential of adjacent epithelia. Science. 2004;303(5659):848-851.

12. Cheng N, et al. Loss of TGF-beta type II receptor in fibroblasts promotes mammary carcinoma growth and invasion through upregulation of TGF-alpha-, MSP- and HGF-mediated signaling networks. Oncogene. 2005;24(32):5053-5068.

13. He Y, et al. Tissue-specific consequences of cyclin D1 overexpression in prostate cancer progression. Cancer Res. 2007;67(17):8188-8197.

14. Valencia $\mathrm{T}$, et al. Metabolic reprogramming of stromal fibroblasts through p62-mTORC1 signaling promotes inflammation and tumorigenesis. Cancer Cell. 2014;26(1):121-135.

15. Minciacchi VR, et al. MYC mediates large oncosome-induced fibroblast reprogramming in prostate cancer. Cancer Res. 2017;77(9):2306-2317.

16. Fiaschi T, et al. Reciprocal metabolic repro- gramming through lactate shuttle coordinately influences tumor-stroma interplay. Cancer Res. 2012;72(19):5130-5140.

17. Sotgia F, Martinez-Outschoorn UE, Pavlides S, Howell A, Pestell RG, Lisanti MP. Understanding the Warburg effect and the prognostic value of stromal caveolin-1 as a marker of a lethal tumor microenvironment. Breast Cancer Res. 2011;13(4):213.

18. Commisso C, et al. Macropinocytosis of protein is an amino acid supply route in Ras-transformed cells. Nature. 2013;497(7451):633-637.

19. Perera RM, Bardeesy N. Pancreatic cancer metabolism: breaking it down to build it back up. Cancer Discov. 2015;5(12):1247-1261.

20. Koochekpour S, et al. Serum glutamate levels correlate with Gleason score and glutamate blockade decreases proliferation, migration, and invasion and induces apoptosis in prostate cancer cells. Clin Cancer Res. 2012;18(21):5888-5901.

21. Afratis N, et al. Glycosaminoglycans: key players in cancer cell biology and treatment. FEBS J. 2012;279(7):1177-1197.

22. Schröder B. The multifaceted roles of the invariant chain CD74--More than just a chaperone. Biochim Biophys Acta. 2016;1863(6 Pt A):1269-1281.

23. Vaishnavi $A$, et al. Oncogenic and drug-sensitive NTRK1 rearrangements in lung cancer. Nat Med. 
2013;19(11):1469-1472.

24. Taylor BS, et al. Integrative genomic profiling of human prostate cancer. Cancer Cell. 2010;18(1):11-22.

25. Cancer Genome Atlas Research Network. The molecular taxonomy of primary prostate cancer. Cell. 2015;163(4):1011-1025.

26. Davidson SM, et al. Direct evidence for cancer-cellautonomous extracellular protein catabolism in pancreatic tumors. Nat Med.2017;23(2):235-241.

27. Tardito $S$, et al. Glutamine synthetase activity fuels nucleotide biosynthesis and supports growth of glutamine-restricted glioblastoma. Nat Cell Biol. 2015;17(12):1556-1568.

28. Wilson M, Gill SK, MacPherson L, English M, Arvanitis TN, Peet AC. Noninvasive detection of glutamate predicts survival in pediatric medulloblastoma. Clin Cancer Res. 2014;20(17):4532-4539.

29. Cai K, et al. Magnetic resonance imaging of glutamate. Nat Med. 2012;18(2):302-306.

30. Wu C, Huang J. Phosphatidylinositol 3-kinase-AKTmammalian target of rapamycin pathway is essential for neuroendocrine differentiation of prostate cancer. JBiol Chem. 2007;282(6):3571-3583.

31. Qian ZR, et al. Prognostic significance of MTOR pathway component expression in neuroendocrine tumors. JClin Oncol. 2013;31(27):3418-3425.

32. Kalin TV, et al. Increased levels of the FoxM1 transcription factor accelerate development and progression of prostate carcinomas in both TRAMP and LADY transgenic mice. Cancer Res. 2006;66(3):1712-1720.

33. Andersson E, et al. Expression profiling of small intestinal neuroendocrine tumors identifies subgroups with clinical relevance, prognostic markers and therapeutic targets. Mod Pathol. 2016;29(6):616-629.

34. Chan DW, et al. DLX1 acts as a crucial target of FOXM1 to promote ovarian cancer aggressiveness by enhancing TGF- $\beta /$ SMAD4 signaling. Oncogene. 2017;36(10):1404-1416.

35. Szklarczyk D, et al. The STRING database in 2017: quality-controlled protein-protein association networks, made broadly accessible. Nucleic Acids Res. 2017;45(D1):D362-D368.
36. Beltran H, et al. Aggressive variants of castrationresistant prostate cancer. Clin Cancer Res. 2014;20(11):2846-2850.

37. Jin $\mathrm{H}$, et al. Epigenetic silencing of a $\mathrm{Ca}(2+)-$ regulated Ras GTPase-activating protein RASAL defines a new mechanism of Ras activation in human cancers. Proc Natl Acad Sci U S A. 2007;104(30):12353-12358.

38. McLaughlin SK, et al. The RasGAP gene, RASAL2, is a tumor and metastasis suppressor. Cancer Cell. 2013;24(3):365-378.

39. Bechtel W, et al. Methylation determines fibroblast activation and fibrogenesis in the kidney. Nat Med.2010;16(5):544-550.

40. Cairns P, et al. Frequent inactivation of PTEN/ MMAC1 in primary prostate cancer. Cancer Res. 1997;57(22):4997-5000.

41. Gravina GL, et al. Hormonal therapy promotes hormone-resistant phenotype by increasing DNMT activity and expression in prostate cancer models. Endocrinology. 2011;152(12):4550-4561.

42. Cunha GR, Hayward SW, Wang YZ, Ricke WA. Role of the stromal microenvironment in carcinogenesis of the prostate. Int J Cancer. 2003;107(1):1-10.

43. Prins GS, Birch L, Greene GL. Androgen receptor localization in different cell types of the adult rat prostate. Endocrinology. 1991;129(6):3187-3199.

44. Wise DR, Thompson CB. Glutamine addiction: a new therapeutic target in cancer. Trends Biochem Sci. 2010;35(8):427-433.

45. Marshall AD, et al. ASCT2 regulates glutamine uptake and cell growth in endometrial carcinoma. Oncogenesis. 2017;6(7):e367.

46. Shih AY, Erb H, Sun X, Toda S, Kalivas PW, Murphy TH. Cystine/glutamate exchange modulates glutathione supply for neuroprotection from oxidative stress and cell proliferation. J Neurosci. 2006;26(41):10514-10523.

47. Rabinovich S, et al. Diversion of aspartate in ASS1-deficient tumours fosters de novo pyrimidine synthesis. Nature. 2015;527(7578):379-383.

48. Beltran $\mathrm{H}$, et al. Challenges in recognizing treatment-related neuroendocrine prostate cancer. JClin Oncol. 2012;30(36):e386-e389.
49. Huang J, et al. Differential expression of interleukin-8 and its receptors in the neuroendocrine and non-neuroendocrine compartments of prostate cancer. Am J Pathol. 2005;166(6):1807-1815.

50. Aaronson DS, et al. An androgen-IL-6-Stat3 auto crine loop re-routes EGF signal in prostate cancer cells. Mol Cell Endocrinol. 2007;270(1-2):50-56.

51. Fina F, et al. Molecular profile of androgenindependent prostate cancer xenograft LuCaP 23.1. JSteroid Biochem Mol Biol. 2005;96(5):355-365.

52. Tolhurst G, Zheng Y, Parker HE, Habib AM, Reimann F, Gribble FM. Glutamine triggers and potentiates glucagon-like peptide-1 secretion by raising cytosolic Ca2+ and cAMP. Endocrinology. 2011;152(2):405-413.

53. Wang Q, et al. Targeting ASCT2-mediated glutamine uptake blocks prostate cancer growth and tumour development. JPathol. 2015;236(3):278-289.

54. Moore SR, et al. Glutamine and alanyl-glutamine promote crypt expansion and mTOR signaling in murine enteroids. Am J Physiol Gastrointest Liver Physiol. 2015;308(10):G831-G839.

55. Kanayama M, et al. Hyperactive mTOR induces neuroendocrine differentiation in prostate cancer cell with concurrent up-regulation of IRF1. Prostate. 2017;77(15):1489-1498.

56. van Geldermalsen M, et al. ASCT2/SLC1A5 controls glutamine uptake and tumour growth in triple-negative basal-like breast cancer. Oncogene. 2016;35(24):3201-3208.

57. Tuxhorn JA, McAlhany SJ, Dang TD, Ayala GE, Rowley DR. Stromal cells promote angiogenesis and growth of human prostate tumors in a differential reactive stroma (DRS) xenograft model. Cancer Res. 2002;62(11):3298-3307.

58. Haldar S, et al. Histone deacetylase inhibitors mediate DNA damage repair in ameliorating hemorrhagic cystitis. Sci Rep. 2016;6:39257.

59. Stark HJ, Baur M, Breitkreutz D, Mirancea N, Fusenig NE. Organotypic keratinocyte cocultures in defined medium with regular epidermal morphogenesis and differentiation. J Invest Dermatol. 1999;112(5):681-691. 